\title{
Die Aufgabenteilung zwischen Mediziner und Rechtsanwender
}

\section{Zusammenfassung des Urteils}

Das Bundesgericht hat im Juni 2014 ein Urteil in einem Invalidenversicherungsfall gefällt, das in den Medien diskutiert wurde. Es soll nicht auf den Einzelfall und die Abgrenzung von (gemäss Gerichtspraxis grundsätzlich nicht invalidisierender) Schmerzstörung und depressiver Störung eingegangen werden. Anlass für den Artikel sind Feststellungen des Bundesgerichts, die in der Ärzteschaft zu Missverständnissen führen könnten. Das Bundesgericht beschreibt in seinem Urteil in Erwägung 3.2. die Verteilung der Aufgaben und Kompetenzen im Rahmen der Invaliditätsbemessung wie folgt: «Sache des (begutachtenden) Mediziners ist es erstens, den Gesundheitszu- stand zu beurteilen und wenn nötig seine Entwicklung im Laufe der Zeit zu beschreiben, d. h. mit den Mitteln fachgerechter ärztlicher Untersuchung unter Berücksichtigung der subjektiven Beschwerden die Befunde zu erheben und gestützt darauf die Diagnose zu stellen. [...] Bei der Folgenabschätzung der erhobenen gesundheitlichen Beeinträchtigungen für die Arbeitsfähigkeit kommt der Arztperson hingegen keine abschliessende Beurteilungskompetenz zu.» Und das Bundesgericht führt weiter aus: «Denn zwischen ärztlich gestellter Diagnose und Arbeitsunfähigkeit - und zwar sowohl bei somatisch dominierten als auch psychisch dominierten Leiden - besteht keine Korrelation.»

\section{Renato Marelli}

Präsident der Schweizerischen Gesellschaft für Versicherungspsychiatrie SGVP
Korrespondenz:

Dr. med. Renato Marelli Schweiz. Gesellschaft für Versicherungspsychiatrie SGVP Leonhardsstrasse 16 CH-4051 Base

\section{Versicherungsmedizinischer Kommentar}

Das Bundesgericht hat sich im erwähnten Fall ausführlich zur Aufgabenteilung zwischen Mediziner und Rechtsanwender bei der Bemessung der Arbeitsunfähigkeit geäussert. Verschiedentlich fragten sich Kollegen - und auch in der Presse wurde darauf eingegangen - welche Kompetenzen ihnen nun bei der Festlegung der Arbeitsunfähigkeit denn wirklich zuständen. Aus diesem Grund sei von versicherungsmedizinischer Seite ein Kommentar gestattet.

\section{Arbeitsunfähigkeit im Bereich der Invaliditätsbemessung}

Zunächst sei festgestellt, dass besagtes Urteil des Bundesgerichts im Rahmen einer Invaliditätsbemessung ergangen ist. In einem solchen Fall steht nicht so sehr eine akut aufgetretene, sondern eine langdauernde Arbeitsunfähigkeit zur Diskussion. Berücksichtigt wird nicht nur die Arbeitsunfähigkeit im bisherigen Beruf oder Aufgabenbereich, sondern auch in einer anderen Tätigkeit bzw. in einem anderen Beruf oder einem anderen Aufgabenbereich. Bei der Invaliditätsbemessung geht es darum, von medizinischer Seite sowohl die Leistungsressourcen als auch die funktionellen Defizite und Einschränkungen darzustellen, aus welchen eine Leistungseinbusse resultiert. Darüber hinaus gilt es, die Therapierbarkeit abzuschätzen respektive eine entsprechende Prognose zu treffen.

Dem aufmerksamen Leser fällt bei den Feststellungen des Bundesgerichts auf, dass ein für die Bemessung der Arbeitsfähigkeit ebenso entscheidender wie unerlässlicher Schritt nach der Stellung der Diagnose keine Erwähnung findet: Die begutachtende Person hat nämlich auszuführen, welche körperli- chen und/oder psychischen Funktionen in welchem Umfang und in welcher Art und Weise eingeschränkt sind und daraus abzuleiten, welche Aktivitäten der versicherten Person nicht oder nur in eingeschränktem Umfang auszuüben noch möglich sind. Es ist schade, dass die Erwähnung dieser Etappe im besagten Bundesgerichtsurteil unerwähnt blieb und somit vor allem bei Personen, die nicht häufig mit medizinischen Gutachten für die Invalidenversicherung befasst sind, zu Fehldeutungen führen kann.

Die explizite Erwähnung dieses Schrittes im Urteil wäre aus drei Gründen nützlich gewesen, um möglichen Missverständnissen vorzubeugen:

Erstens ist es dem Rechtsanwender nur möglich, seine genuine Aufgabe der Folgenabschätzung für die Arbeitsfähigkeit im Hinblick auf die Frage einer Erwerbsunfähigkeit zu erfüllen, wenn er erkennen kann, dass ein zu diagnostizierender gesundheitlicher Schaden vorliegt und vor allem wie und weshalb die Arztperson zu ihrer Einschätzung der Arbeitsfähigkeit gekommen ist. Erst die Abschätzung der Zumutbarkeit ermöglicht in einem darauffolgenden weiteren Schritt, die zuvor erfolgte medizinische Einschätzung aus rechtlicher Sicht zu beurteilen, d. h. nachzuvollziehen oder zu korrigieren. Auch bei divergierenden Angaben zur Arbeitsunfähigkeit was durchaus in der Natur der medizinischen Beurteilung liegen kann - obliegt es dem Rechtsanwender, diese rechtlich zu würdigen.

Zweitens würde die Aussage des Bundesgerichts «Zwischen ärztlich gestellter Diagnose und Arbeitsunfähigkeit (...) besteht keine Korrelation» mit der Ergänzung, dass gesundheitlich bedingte Funktions- 
und Aktivitätseinschränkungen durch die Arztperson zu erläutern sind, auch für den gutachterlich nicht geschulten Leser verständlich. Es gibt nämlich zweifellos somatische wie psychiatrische Diagnosen, bei denen ein Laie davon ausgehen kann, dass die Arbeitsfähigkeit der betroffenen Person deutlich beeinträchtigt ist. Bei einem akuten Herzinfarkt korrelieren Krankheit und Arbeitsunfähigkeit sehr eng. Ein ebensolches Beispiel ist etwa ein paranoid-halluzinatorisches Syndrom im Rahmen einer Schizophrenie. Tatsache bleibt dennoch auch bei diesen Beispielen, dass sich die Attestierung der Arbeitsunfähigkeit erst durch die kausale Abfolge von Befundkonstellation im Rahmen der Diagnose mit sich daraus ergebender Störung der körperlichen oder psychischen Funktionen und sich wiederum daraus ableitender Einschränkung der Aktivitäten und der Partizipation im Arbeitsprozess ergibt. Besonders in den Fällen, die im Rahmen der Invaliditätsbemessung zur Begutachtung kommen und bei denen, mit welchen sich der Richter auseinandersetzt, ist es somit unerlässlich, dass ärztlicherseits eine Beurteilung der Funktionen und Aktivitäten vorliegt. In solchen Fällen ist der Zusammenhang zwischen der Befundkonstellation und der möglichen Arbeitsunfähigkeit praktisch nie derart eng wie in den oben genannten Beispielen, das heisst es besteht tatsächlich keine (direkte) Korrelation zwischen Diagnose und Arbeitsunfähigkeit. Das Bundesgericht zitiert dazu eine Studie, die pars pro toto diesem Umstand Rechnung trägt [1].

Drittens wäre ein solcher Hinweis im Urteil auch ein Appell an diejenigen Ärztinnen und Ärzte gewesen, die sich die Beschreibung der Einschränkungen der Funktionen und Aktivitäten bei der Attestierung einer Arbeitsunfähigkeit noch nicht routinemässig zu eigen gemacht haben. Dieser Appell soll deshalb an dieser Stelle nachgeholt werden.

\section{Arbeitsunfähigkeit im Rahmen der Taggeldversicherung oder im Attest für den Arbeitgeber}

In den Medien wurde noch die Frage aufgeworfen nach allfälligen Folgen des erwähnten Bundesge- richtsurteils für ärztliche Atteste im Bereich der Taggeldversicherung. Auf solche bezieht sich das Urteil jedoch klar nicht. Im Bereich der Taggeldversicherung obliegt es der Arztperson, nach bestem Wissen und Gewissen die Arbeitsfähigkeit abschliessend zu beurteilen. Eine solche Beurteilung kann durch den Arbeitgeber bzw. die Taggeldversicherung selbstverständlich angefochten werden, wenn sie nicht nachvollziehbar ist oder wenn neue, zuvor nicht bekannte Tatsachen Zweifel an der Beurteilung nach sich ziehen. Doch obliegt es in einem solchen Fall Arbeitgeber bzw. Versicherer, eine erneute ärztliche Beurteilung einzuholen. Erst diese kann die erste medizinische Beurteilung allenfalls erschüttern und/oder korrigieren.

1 Klipstein, Michel, Läubli et al. Do MRI findings correlate with mobility tests? Eur Spine. 2007; 803-11.

\section{Zusammenfassung und Erwartungen} Zusammengefasst enthält das Urteil nichts, was nicht schon jetzt im Rahmen der Invaliditätsbemessung bekannt gewesen wäre. Dennoch sei aus versicherungsmedizinischer Sicht im Hinblick auf die Verbesserung der Zusammenarbeit je eine Erwartung an die medizinischen Fachpersonen und eine an die Rechtsanwender ausgedrückt:

Bei der Bestimmung der Arbeitsunfähigkeit sind Ärztinnen und Ärzte gefordert, sich neben der medizinisch-diagnostischen Abklärung vermehrt auch zu Funktionsstörungen, Leistungseinbussen und Ressourcen zu äussern.

Umgekehrt darf von Rechtsanwendern erwartet werden, dass sie aus rechtlicher Sicht sorgfältig begründen, warum sie von der ärztlichen Einschätzung der Arbeitsunfähigkeit abweichen bzw. zu anderen Schlüssen kommen.

In diesem Sinne soll die Aufgabenteilung zwischen Medizin und Recht im Rahmen der zur Frage stehenden Bemessung der Arbeitsunfähigkeit erspriesslich sein.

\section{Hanspeter Kuhn}

Fürsprecher, Leiter Abteilung Rechtsdienst FMH

\footnotetext{
Korrespondenz: Hanspeter Kuhn FMH-Rechtsdienst Postfach 18 CH-3000 Bern 15 Tel. 0313591111 Fax 0313591112 lex[at]fmh.ch
}

\section{Juristischer Kommentar}

Dr. med. Renato Marelli ist beizupflichten, dass eine wichtige Aufgabe des ärztlichen Gutachters darin besteht, auszuführen, «welche körperlichen und/ oder psychischen Funktionen in welchem Umfang und in welcher Art und Weise eingeschränkt sind, und daraus abzuleiten, welche Aktivitäten der versicherten Person nicht oder nur in eingeschränktem Umfang auszuüben noch möglich sind». - «Lehre und Rechtsprechung gehen beim Begriff der Arbeitsunfähigkeit von einer körperlichen, geistigen oder psychischen Einschränkung aus, die zur bedingten, vollständigen oder teilweisen Unfähigkeit führt, im bisherigen Beruf bzw. Aufgabenbereich zumutbare Arbeit zu leisten [1]. Zur Sachverhaltserstellung gehört daher die Einschätzung und Würdigung von Leistungsfähigkeit und Stellenprofil durch eine hierzu befähigte Fachperson. Die medizinisch-theoretische Arbeitsfähigkeit [...] ist grundsätzlich durch den Arzt festzulegen, da es um die körperlichen und geistigen Fähigkeiten geht.» [2] Dabei hat der Gut- 
achter alle Befunde nach dem Stand der medizinischen [3] Wissenschaft sorgfältig zu gewichten. Dass für die korrekte medizinische Beurteilung nicht immer primär auf aus Laiensicht sogenannt objektive Untersuchungsmethoden wie das MRI abgestellt werden kann, zeigt gerade die vom Bundesgericht im Urteil zitierte Studie auf. Eine Fixierung auf Laborbefunde oder bildgebende Verfahren entspräche einem reduktionistischen Verständnis medizinischer Wissenschaft. Auch der Gesetzgeber hat explizit ein umfassenderes Medizinverständnis in der Schweizer Rechtsordnung verankert. Absolvierende des Medizinstudiums «verstehen gesundheitliche Probleme ganzheitlich und erfassen dabei insbesondere die physischen, psychischen, sozialen, rechtlichen, ökonomischen, kulturellen und ökologischen Faktoren und Auswirkungen und beziehen diese in die Lösung der gesundheitlichen Probleme auf individueller und Gemeinschaftsebene ein.» [4]

Aus rechtlicher Sicht erscheint der Satz aus dem Bundesgerichtsentscheid (BGE) «zwischen ärztlich gestellter Diagnose und Arbeitsunfähigkeit - und zwar sowohl bei somatisch dominierten als auch bei psychisch dominierten Leiden - besteht keine Korrelation» zumindest missverständlich. Zu offensichtlich sind die Beispiele, bei denen das Gegenteil zutrifft und ein klarer Zusammenhang zwischen Diagnose und Arbeitsunfähigkeit besteht. Woher kann diese Sprachverwirrung herrühren?

Halten wir zunächst fest: Der Begriff «Arbeitsunfähigkeit» kommt in den gesetzlichen Definitionen für die Invalidenversicherung nicht vor:

«Invalidität ist die voraussichtlich bleibende oder längere Zeit dauernde ganze oder teilweise Erwerbsunfähigkeit.» (Art. 8 Bundesgesetz über den allgemeinen Teil der Sozialversicherung, ATSG).

«Erwerbsunfähigkeit ist der durch Beeinträchtigung der körperlichen, geistigen oder psychi- schen Gesundheit verursachte und nach zumutbarer Behandlung und Eingliederung verbleibende ganze oder teilweise Verlust der Erwerbsmöglichkeiten auf dem in Betracht kommenden ausgeglichenen Arbeitsmarkt.» (Art. 7 ATSG).

Die in Art. 6 ATSG gesetzliche definierte Arbeitsunfähigkeit deckt sodann zwei unterschiedliche Lebenssachverhalte ab:

«Arbeitsunfähigkeit ist die durch eine Beeinträchtigung der körperlichen, geistigen oder psychischen Gesundheit bedingte, volle oder teilweise Unfähigkeit, im bisherigen Beruf oder Aufgabenbereich zumutbare Arbeit zu leisten. Bei langer Dauer wird auch die zumutbare Tätigkeit in einem anderen Beruf oder Aufgabenbereich berücksichtigt.»

Der folgende Formulierungsvorschlag ist deshalb zwar länger, wird aber wohl der Doppelfunktion des Begriffs Arbeitsunfähigkeit im ATSG eher gerecht: «Zwischen ärztlich gestellter Diagnose und Arbeitsunfähigkeit - besteht in der Regel und insbesondere bei langfristigen Arbeitsunfähigkeiten keine direkte Korrelation.»

\section{Literatur}

1 Scartazzini G, Hürzeler M. Bundessozialversicherungsrecht. 4. Aufl. Basel; 2012. S. 59 f.

2 Stolkin P. Was ist Sachverhalt und was ist Recht?. In: Jusletter. 25. August 2014. Randziffer 31.

3 Zutreffend Stolkin, op.cit., Randziffer 81: «... Es ist allein Sache des Gutachters, die wissenschaftlichen Erfahrungssätze zu bestimmen, mit denen er zu einem Resultat zu kommen gedenkt und nicht jene des Gerichts. Freilich hat er seine Erkenntnisse gegenüber dem Gericht nachvollziehbar und für die Juristen verständlich zu schildern. ...»

4 Art. 8 lit. f Medizinalberufegesetz.

\section{Aktuelle}

Forumthemen

Diskutieren Sie mit! Im Forum präsentieren wir regelmässig brisante Themen aus Politik, Ökonomie und Wissenschaft, die das Schweizer Gesundheitswesen betreffen. Bringen Sie Ihre Meinung ein oder kommentieren Sie die Äusserungen Ihrer Kolleginnen und Kollegen. Das Forum finden Sie unter: www.saez.ch/forum/ 